\title{
Research on Assembly Process Modelling Method based Assembly Task Collection
}

\author{
Yang Yunbin ${ }^{1, a^{*}}$, Liao Hongqiang ${ }^{1, b}$ and He Liangli ${ }^{1, c}$ \\ ${ }^{1}$ Institute of Systems Engineering, CAEP, Mianyang Sichuan, China, 621999 \\ ayyb717@163.com, bliaohq@caep.cn, chell@caep.cn
}

\begin{abstract}
Keywords: Hierarchical structure; assembly task; assembly process; virtual assembly
Abstract: Assembly process model illuminating product assembly is the base of assembly simulation and assembly assessment, and assembly process model based ordinal assembly task link can not describe hierarchical structure and parallelism of assembly task. The development actuality of assembly process modelling is simply investigated, and product hierarchy information model is established, and its implementation method is illuminated, and the detailed description of assemlby task is analyzed. According to hierarchical relation of assembly task, assembly process modelling method is achieved based ATCLHS (Assembly Task Collection Link based Hierarchical Structure). Storage method and access method of ATCLHS is established based nested table. An example of assembly process model based assembly task collection is illuminated by ATCLHS.
\end{abstract}

\section{Introduction}

In order to making assembly assessment and assembly optimization in design stage, the questions of assembly assessment, assembly optimization and assembly validation are solved by virtual assembly technique based DMU (Digital Mock-Up). Assembly process model describes the whole process of product assembly simulation, and it records all information of assembly simulation process. The integrative assembly process is the base of assembly analysis and assembly validation, and it guarantees the validity and integrality of virtual assembly information. At present, assembly process modelling method is widely studied, but assembly resource, assembly person, and assembly environment is rarely considered. The existing assembly process model cannot describe hierarchical structure and parallelism of assembly task, so it lead to the difference of between actual assembly process with virtual assembly process, and engineering application of virtual assembly is restrained. In reference [1], product assembly process is described by the directional sequence assembly task link, the relation of time and order between assembly tasks with assembly restriction is expressed. Sequence assembly process can be described by the model, but hierarchical relation can not be described, and parallelism of assembly task can not be described either. In reference [2], a product assembly process modelling method based on hierarchy chain technique was proposed; assembly hierarchical structure can be expressed by the model. Because the node of hierarchical link is an assembly task, parallelism of assembly task can not be described.

\section{Product hierarchy information model}

In virtual assembly process, product hierarchy information model is the base of assembly simulation. Product hierarchy information model is established by analyzing the demand of produt model in simulation process [3]. Fig. 1 depicts product hierarchy information model. 


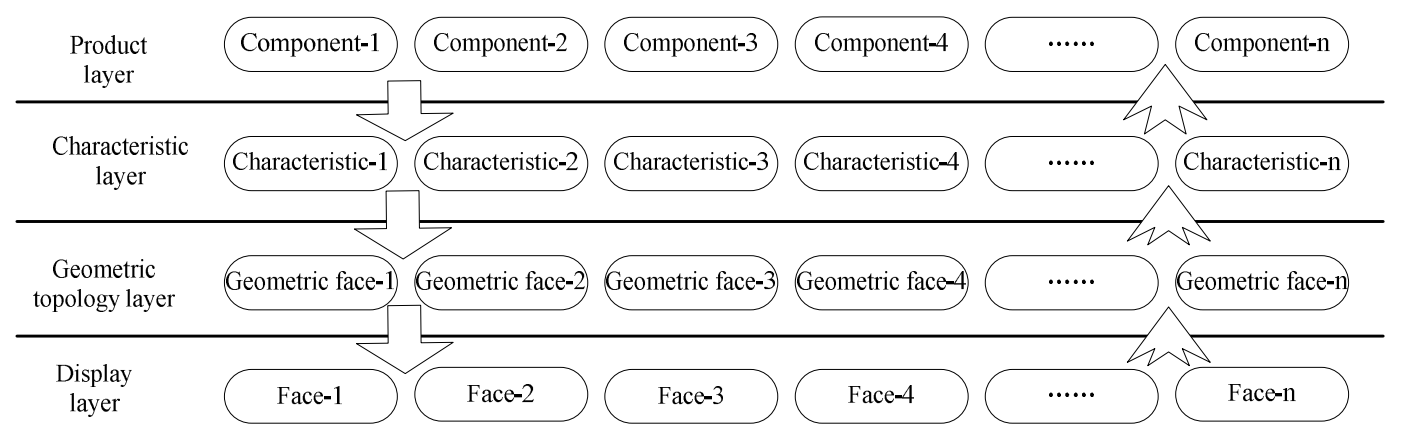

Fig. 1 Product hierarchy information model

Based the characteristic of actual assembly process, information need of product virtual assembly model is analyzed in virtual assembly process. Virtual assembly model based product hierarchy information model is established, it includes product geometrical information, product physically information and product assembly information, and the representation method is founded. Part topology information, part geometrical information, part assembly mating information are obtained from CAD model by developing, part physically information and part assembly characteristic are defined by interactive method. Fig. 2 depicts the way of virtual assembly modelling.

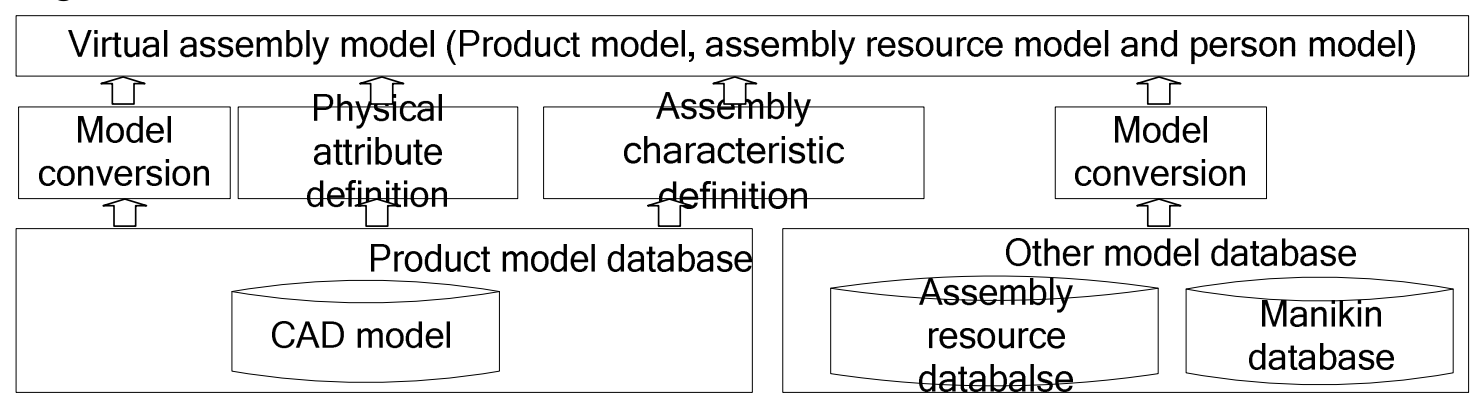

Fig. 2 The way of virtual assembly modelling

\section{Assembly task description}

Assembly process is a sequential collection of a series of assembly tasks, and assembly task is a basal unit of assembly process, it includes a series operation process in order to finish one assembly unit in virtual environment. Manipulation process of a typical assembly task includes specifying assembly benchmark, picking up part, moving part, releasing part, and so on. Assembly task description includes assembly task identifier, assembly sequence, task type, task object, assembly path, assembly time, and so on, and the whole description of assembly task is shown in Fig. 3.

\begin{tabular}{|c|c|c|c|c|c|c|c|c|c|c|c|c|c|c|}
\hline \multicolumn{15}{|c|}{ Assembly task } \\
\hline & demb & task & & mbly & Tas & type & & Tas & $\checkmark$ & & & mbly & & $\begin{array}{l}\text { m } \\
\text { embly } \\
\text { me }\end{array}$ \\
\hline 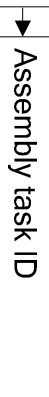 & 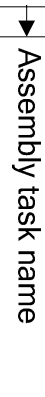 & 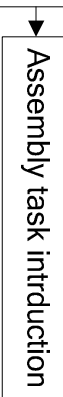 & 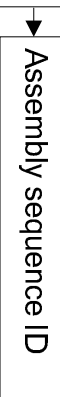 & 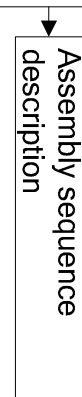 & 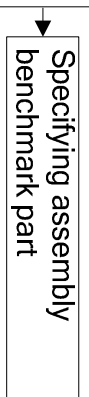 & 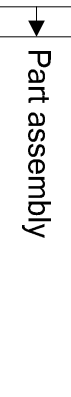 & $\begin{array}{l}\downarrow \\
\stackrel{D}{7}\end{array}$ & 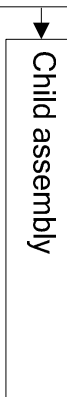 & 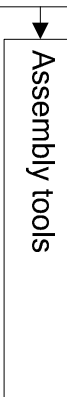 & 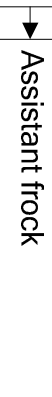 & 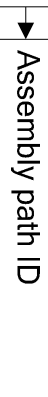 & 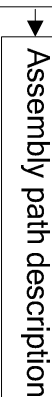 & 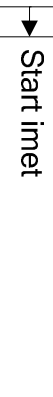 & 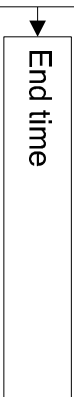 \\
\hline
\end{tabular}

Fig. 3 Assembly task decription 
Assembly task is introduced by assembly task identifier, and assembly task uniqueness is guaranteed by assembly task ID. Assembly sequence is an ordered collection of a series of manipulation, and it is identified by assembly sequence ID, assembly sequence of part in virtual environment is recorded by linked list form, every node in the linked list can be described by nether data structure:

struct sequence_node $\{$ int id;

part current_part;

path move_path; //assembly sequence ID

//current assembly unit

//assembly path of current assembly unit

//next assembly node \}

Task type includes specifying assembly benchmark part and part assembly, Specifying assembly benchmark part don't need define assembly restriction relation. Task object includes assembly cell, assembly tools, assistant frock, and so on, and its interrelated information is obtained by product hierarchy information model. Assembly path records manipulation path of task object, and it illuminates locomotive track of part and assembly tool in virtual assembly environment, and it is identified by assembly path ID. The position and orientation of part and assembly tool are recorded by the discrete node in virtual assembly process, and these nodes are organized by linked list form, and the assembly path of part and assembly tool is gained based the nodes. Every node in the linked list can be described by nether data structure:

struct path_node\{ orientation part_orientation; //part orientation

$\begin{array}{ll}\text { position part_position; } & / / \text { part position } \\ \text { path_node next_node; } & / / \text { next node }\end{array}$

Assembly time include start time and end time, if assembly time is only described by the need time of assembly manipulation, start time and end time of assembly manipulation can not be expressed.

\section{Assembly process model based assembly task collection}

Assembly task collection link based hierarchical structure. Assembly process is made up a series of assembly task in virtual assembly simulation. Assembly task of complex product includes multilayer structure, such as system layer, component layer, part layer, and etc. the upper layer task is depended on the under layer task. Thing of multilayer structure of complex product and parallelism of assembly task, Assembly process of complex product is described by ATCLHS, Fig. 4 depicts ATCLHS. In Fig. $4, \mathrm{~S}_{k}$ is the $\mathrm{k}$ task collection of system layer, $\mathrm{C}_{o}$ is the o task collection of component layer, $\mathrm{P}_{\mathrm{s}}$ is the $\mathrm{s}$ task collection of part layer, $\mathrm{T}_{i}$ is the $\mathrm{i}$ task of one task collection. $\mathrm{S}_{k} \mathrm{~T}_{i}$ is the $\mathrm{i}$ task of the $\mathrm{k}$ task collection in system layer, and $\mathrm{P}_{s} \mathrm{~T}_{r}$ is the $\mathrm{r}$ task of the $\mathrm{s}$ task collection in part layer.

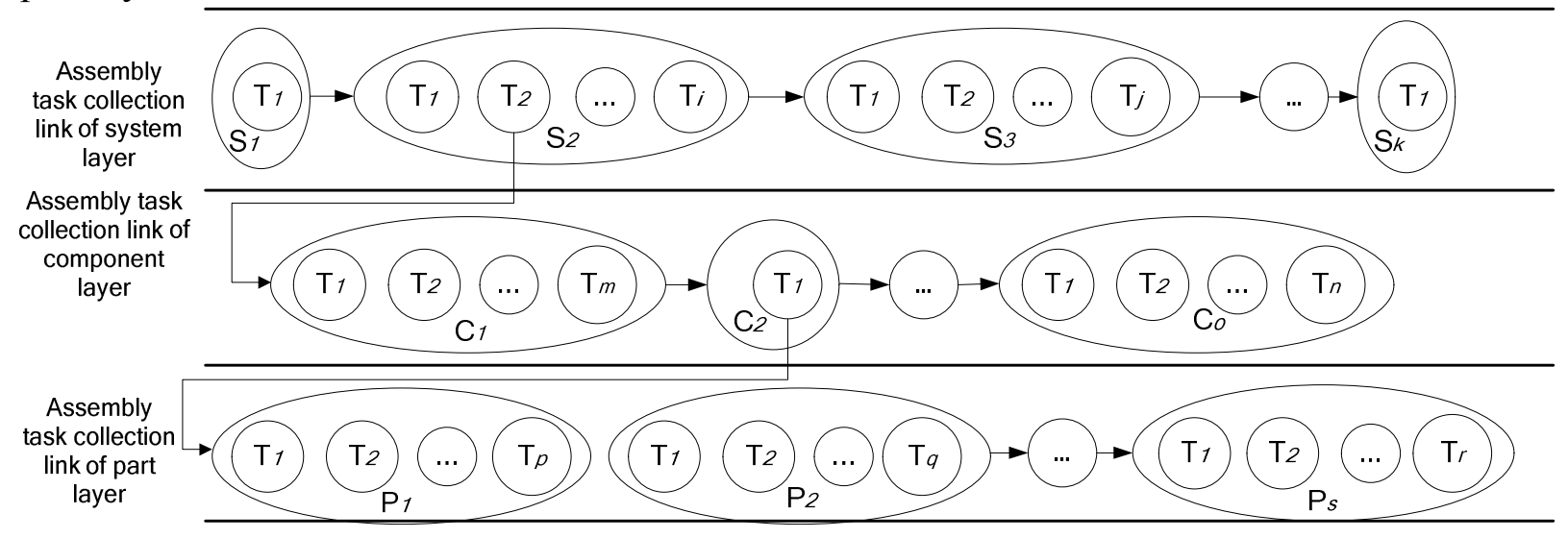

Fig. 4 Assembly task collection link based hierarchical structure

Task collection node of task collection link is described by nether data structure:

struct task_collection_node $\{$ int id;

$/ /$ task collection ID 
int task_level; $\quad / /$ task collection is situated in task layer bool is_parallel; // if task collection has parallel task

bool is_finish; // task collection finish state task_collection_node* next_node; //next node of task collection task_collection tasks; $\quad \overline{/}$ task collection includes tasks \}

Task collection is made up of a series of assembly task ID, and assembly task ID represents the corresponding assembly task. If next_node of task collection node is null, it means the task collection is the last task collection of the layer.

Task node of task collection is described by nether data structure:

struct task_node $\{$ int id; //task ID

int task_level; $\quad / /$ task is situated in task layer
bool is_parallel; $\quad / /$ if task is a parallel task
bool is_finish; $\quad / /$ task finish state
task_collection_node* child_node;

If child node of assembly task is null, it means the assembly task not has the under layer assembly task collection link.

There are many advantages describing product assembly process by ATCLHS:

(1) Hierarchical structure and parallelism of assembly task: ATCLHS can describe parallelism manipulation. Because every node of assembly task collection link is an assembly task collection, ATCLHS sustains parallelism manipulation of assembly tasks in the same layer. Based multilayer structure, it guaranteed the under layer assembly tasks can be started at the same time, and then the upper layer task are carried out. ATCLHS embodies layer structure of assembly process.

(2) Dynamic characteristic: ATCLHS can express dynamic process information in virtual assembly simulation process. Based dynamic process information, the designer can analyze product dynamic behavior again and again, and much information is provided for the next phase of virtual assembly. Specially, assembly order can be chosen by the operator based their knowledge for parallel assembly task, and dynamic process information of assembly task can be recorded, the different assembly flow can be assessed base these information.

(3) Roll back: Because assembly time can be considered in assembly task description, and ATCLHS is established based assembly task collection, roll back based assembly time and roll back based assembly task can be all sustained. The different roll back can be chosen for simulation requirement, so the agility of roll back can be guaranteed, and the efficiency can be enhanced.

ATCLHS implementation. In order to realize storage and access of assembly task collection link, nested table technique is adopted. A nested table is a table stored within the structure of another table. A nested table is represented in the father table as a special column that has a data type of table, for any particular row, this kind of column contains selected rows from the child table that pertain to the father table. It is realized that child table information is directly recorded in father table by nested table technique. Father table can directly access child table record, so the efficiency of data access is higher ${ }^{[4]}$. For assembly task collection link of system layer in Fig. 4, storage and access of it can be realized by the nested table in Fig. 5. For assembly task collection $\mathrm{S}_{2}$, its task collection field is a nested table, it is composed of many rows, these rows include $\mathrm{S}_{2} \mathrm{~T}_{1}, \mathrm{~S}_{2} \mathrm{~T}_{2}, \mathrm{~S}_{2} \mathrm{~T}_{i}$, and so on. All tasks of assembly task collection $\mathrm{S}_{2}$ can be accessed by the nested table, the detailed of all assembly tasks can be gained in assembly task table by assembly task ID. By the same way, assembly task collection link of component layer and component layer can be achieved. 
Task colltection table

(father table)

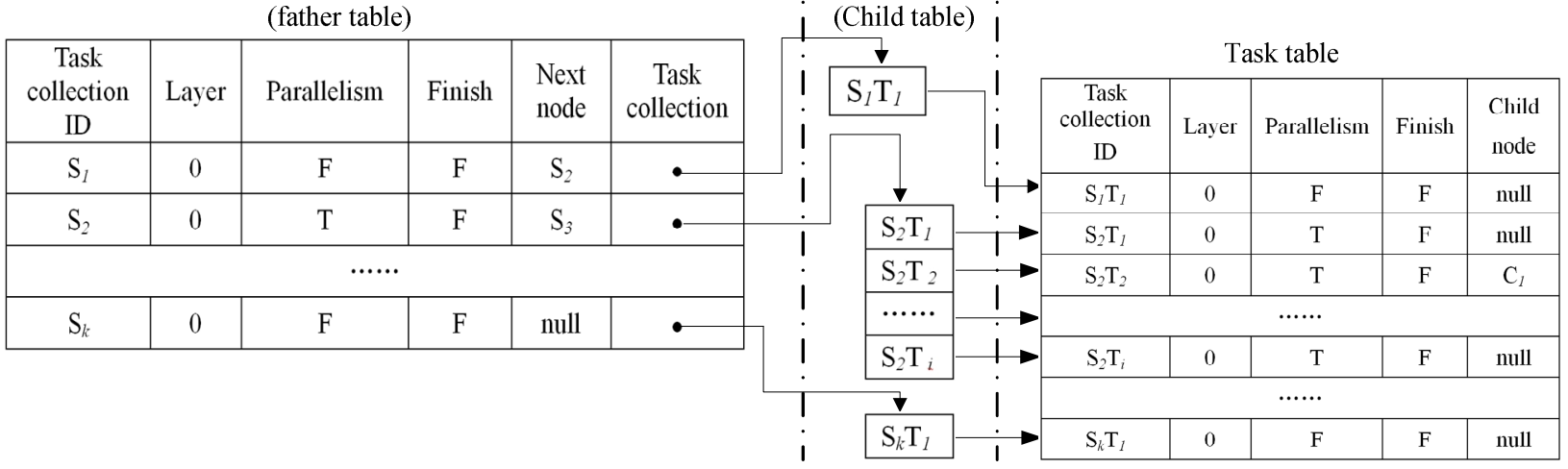

Fig. 5 Storage and access of assembly task collection link in system layer

Application examples. Virtual reality environment includes BARCO Galaxy DLP projection, PowerWall display system, DELL T7500 workstation, Quadro Plex visual server and VR device. Virtual reality environment is an initiative immersion environment with three display channels, and VR device includes CrystalEyes glasses, FOB and neowand [5]. The location and direction of neowand are gained by FOB, the direction operator and system control are realized by neowand that is an input and control device, virtual assembly simulation is realized by this way. The application of assembly process of ATCLHS is illuminated by assembly process with two hierarchical structures, its assembly task collection link is depicted by Fig. 6, and assembly task collection and assembly task of component layer are detailedly described by from Table 1 to Table 4 .

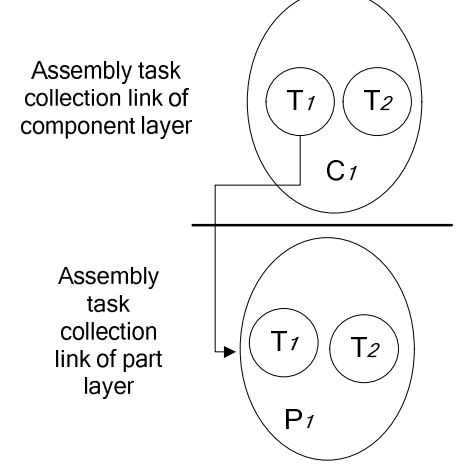

Fig. 6 Task collection link

Table 1 Assembly task collection table of component layer

\begin{tabular}{|c|c|c|c|c|c|}
\hline Task collection ID & Layer & Parallelism & Finish & Next node & Task collection \\
\hline C1 & 0 & T & F & null & C1T1, C1T2 \\
\hline
\end{tabular}

Table 2 Assembly task table of component layer

\begin{tabular}{|c|c|c|c|c|}
\hline Task ID & Layer & Parallelism & Finish & Child node \\
\hline C1T1 & 0 & T & F & P1 \\
\hline C1T2 & 0 & T & F & null \\
\hline
\end{tabular}

Table 3 Assembly task collection table of part layer

\begin{tabular}{|c|c|c|c|c|c|}
\hline Task collection ID & Layer & Parallelism & Finish & Next node & Task collection \\
\hline $\mathrm{P}_{1}$ & 1 & $\mathrm{~T}$ & $\mathrm{~F}$ & null & $\mathrm{P}_{1} \mathrm{~T}_{1}, \mathrm{P}_{1} \mathrm{~T}_{2}$ \\
\hline
\end{tabular}

Table 4 Assembly task table of part layer

\begin{tabular}{|c|c|c|c|c|}
\hline Task ID & Layer & Parallelism & Finish & Child node \\
\hline $\mathrm{P}_{1} \mathrm{~T}_{1}$ & 1 & $\mathrm{~T}$ & $\mathrm{~F}$ & null \\
\hline $\mathrm{P}_{1} \mathrm{~T}_{2}$ & 1 & $\mathrm{~T}$ & $\mathrm{~F}$ & null \\
\hline
\end{tabular}

$\mathrm{C}_{I} \mathrm{~T}_{1}$ : Component II is installed on the fixing panel

$\mathrm{C}_{I} \mathrm{~T}_{2}$ : Component $\mathrm{I}$ is installed on the fixing panel

$\mathrm{P}_{l} \mathrm{~T}_{l}$ : Part II is installed on the component II

$\mathrm{P}_{1} \mathrm{~T}_{2}:$ Part III is installed on the component II 
When the fixing panel is chosen by neowand, and it is specified to assembly benchmark part. According as assembly process model based ATCLHS, it is prompt that $\mathrm{C}_{l} \mathrm{~T}_{1}$ is first finished by virtual assembly system, it means assembly task of part layer must be first finished. Fig. 7(a) shows this assembly process. When component II is chosen by neowand, and it is designated to assembly benchmark part. $\mathrm{P}_{1} \mathrm{~T}_{1}$ and $\mathrm{P}_{1} \mathrm{~T}_{2}$ may be carried out by the parallelism mode or the out-of-order serial mode. Here, the operator can choose the part that is first assembled. Fig. 7(b) shows the process of installing part II after installing part III is finished. Fig. 7(c) shows the simulation result of component II is already installed on the fixing panel.

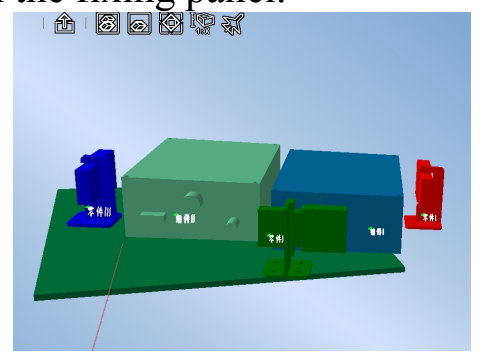

(a) Intelligent tip

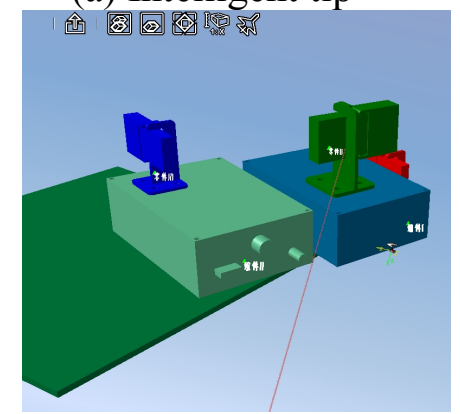

(b) The process of installing part II

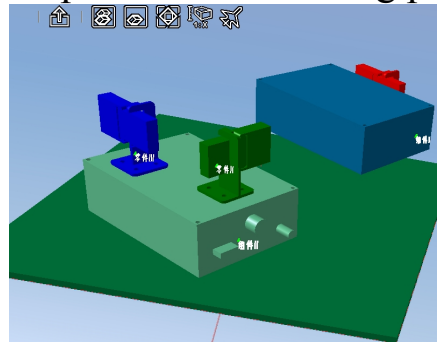

(c) The result of installing component II

Fig. 7 Assembly simulation example based ATCLHS

\section{Conclusion}

Assembly process modelling method based assembly task collection is established aiming at hierarchical structure and parallelism of assembly task can not be described, and Storage and access of assembly process model based ATCLHS can be achieved by nested table technique. By the method, hierarchical structure and parallelism of assembly task can be embodied, and the dynamic information of assembly process can be described, and roll back method of assembly process based assembly time and assembly task is sustained. Assembly process modelling method based assembly task collection is a base of concurrent assembly simulation.

\section{References}

[1] J.R. Tan, Z.Y. Liu, S.Y. Zhang. Roll Back of Assembly Process in Virtual Environment, Computer-aided Design \& Computer Graphics, Vol.15, No.11, 2003

[2] W.W Hou, J.H. Liu, etc. Product assembly process modeling method based on hierarchy chain technique. Computer Integrated Manufacturing Systems, Vol.15, No.8, 2009 
[3] Z.Y. Liu, J.R. Tan, S.Y. Zhang. The Multi-Level Representation of Product Information for Virtual Assembly. Journal of Computer-aided Design \& Computer Graphics, Vol.13, No.3, 2001

[4] Y.B. Yang. Design \& Implementation of the Air Space Management and Evaluation System. Chengdu: Sichuan University, 2003

[5] L.L. He, F.Y. Wei, F.J. Wang. Human-computer interaction in virtual layout/assembly environment. Journal of Machine Design, Vol.27 No.5, 2010 